\title{
Morphology Evolution of Mn-Si Composition Gradient Micro/Nanomaterials Prepared by Oxygen Assisted Chemical Vapor Deposition
}

\author{
Siwei Tang $(\mathbb{D}$ \\ State Key Laboratory of Powder Metallurgy, Central South University, Changsha 410083, China \\ Correspondence should be addressed to Siwei Tang; siweitang@csu.edu.cn
}

Received 14 September 2017; Revised 27 November 2017; Accepted 21 December 2017; Published 18 January 2018

Academic Editor: Miguel A. Garcia

Copyright (C) 2018 Siwei Tang. This is an open access article distributed under the Creative Commons Attribution License, which permits unrestricted use, distribution, and reproduction in any medium, provided the original work is properly cited.

\begin{abstract}
The micro/nanostructure of manganese silicide ( $\mathrm{Mn}-\mathrm{Si}$ ) compounds with various morphologies (nanowires, films, particles, and polyhedron shape structure) has been synthesized through oxygen assisted chemical vapor deposition by changing the stacking geometry of manganese powder. Polyhedrons prepared in the Mn-Si contact area were identified to be chemical composition gradient functionally graded materials which were verified by analyzing atomic ratio of $\mathrm{Mn} / \mathrm{Si}$ from top to bottom. Evolution of morphology greatly depended on the stacking shape correlated distance from precursor to the substrate, resulting in distinctive growth mechanisms. Main structures on the substrate have been verified to be $\mathrm{Mn}_{5} \mathrm{Si}_{3}$ and $\mathrm{Mn}_{4} \mathrm{Si}_{7}$ with different $\mathrm{Mn}$ stacking in bumps comparing to sole $\mathrm{Mn}_{4} \mathrm{Si}_{7}$ with flat surface.
\end{abstract}

\section{Introduction}

Recently, manganese silicide nanostructures which have unusual magnetic properties are considered to be potential candidates in spintronic applications $[1,2]$. Among them, the most heavily studied phase is $\mathrm{Mn}-\mathrm{Si}$ which undergoes transitions from helimagnetic to magnetic skyrmion phase near $30 \mathrm{~K}$ [3-5]. Skyrmion shows a great potential to be used as a computer device with a much faster computing speed, larger storage capability, and much less energy consumed $[6,7]$. The $\mathrm{Mn}_{4} \mathrm{Si}_{7}$ as a weak itinerant system which orders below $40 \mathrm{~K}$ nanowires has several application as optoelectronic material and thermoelectric material [8-11]. With a hexagonal structure, $\mathrm{Mn}_{5} \mathrm{Si}_{3}$ is another interesting material which has the potential for a high magnetocrystalline anisotropy with novel spin-electronic properties, while the nanocrystal behaved like a ferromagnet due to size effect [12-14]. A diluted magnetic semiconductor $\mathrm{Mn}_{x} \mathrm{Si}_{1-x}(x=0.03-0.05)$ showed a ferromagnetic transition point at $250 \mathrm{~K}$ with a method to manipulate the charge and spin $[15,16] . \mathrm{Mn}_{3} \mathrm{Si}$ was studied to find out the unique electronic and magnetic properties $[17,18] . \mathrm{Mn}_{5} \mathrm{Si}_{2}$ which has a ferromagnetic ordering with a $T_{C}$ of $300 \mathrm{~K}$ [19] does have a potential in the microelectronics.

\section{Methods}

The chemical vapor deposition (CVD) system was set up based on a single zone Lindberg tube furnace with maximum temperature of $1200^{\circ} \mathrm{C}$. High purity Argon gas (99.999\%) was used as the carrier gas. The system allows precise control of growth temperature, gas flow rate, gas pressure, and distance from substrate to precursor. In this home designed CVD system, temperature was set to $850^{\circ} \mathrm{C}$ when flow rate of $\mathrm{Ar}$ gas was varied from 140 to $300 \mathrm{sccm}$. The pressure was always kept at 760 Torr and oxygen was brought into the system for not fully sealing the tube intentionally. A clean Si (001) wafer was placed above the Mn powder as substrate inside a ceramic crucible located in the center of the tube. The Mn powder (99.9\%) was purchased from CHANGSHA TIANJIU Company. The Mn powder was laying in different shape, such as flat, cone, and multiple-cones. The silicon wafer (001) was purchased from HEFEI KEJING Materials technology CO., LTD. Silicon (100) chips were cut into $1 \mathrm{~cm} \times 1 \mathrm{~cm}$ in size, and they are put in deionized water sonicating for $10 \mathrm{~min}$. Then they are washed by ethanol sonicating for 10 mins and dried with $\mathrm{N}_{2}$ gas.

Scanning electron microscope (FEI Nano230) (SEM) equipped with an energy dispersive X-ray spectrometer 


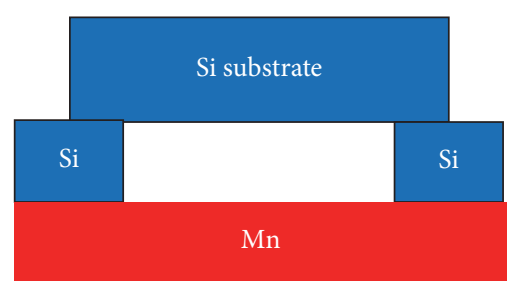

(a)

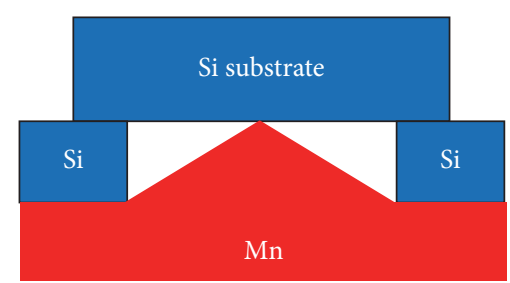

(b)

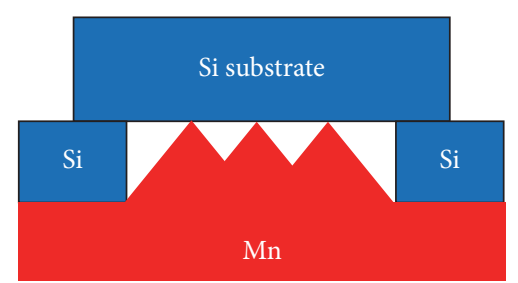

(c)

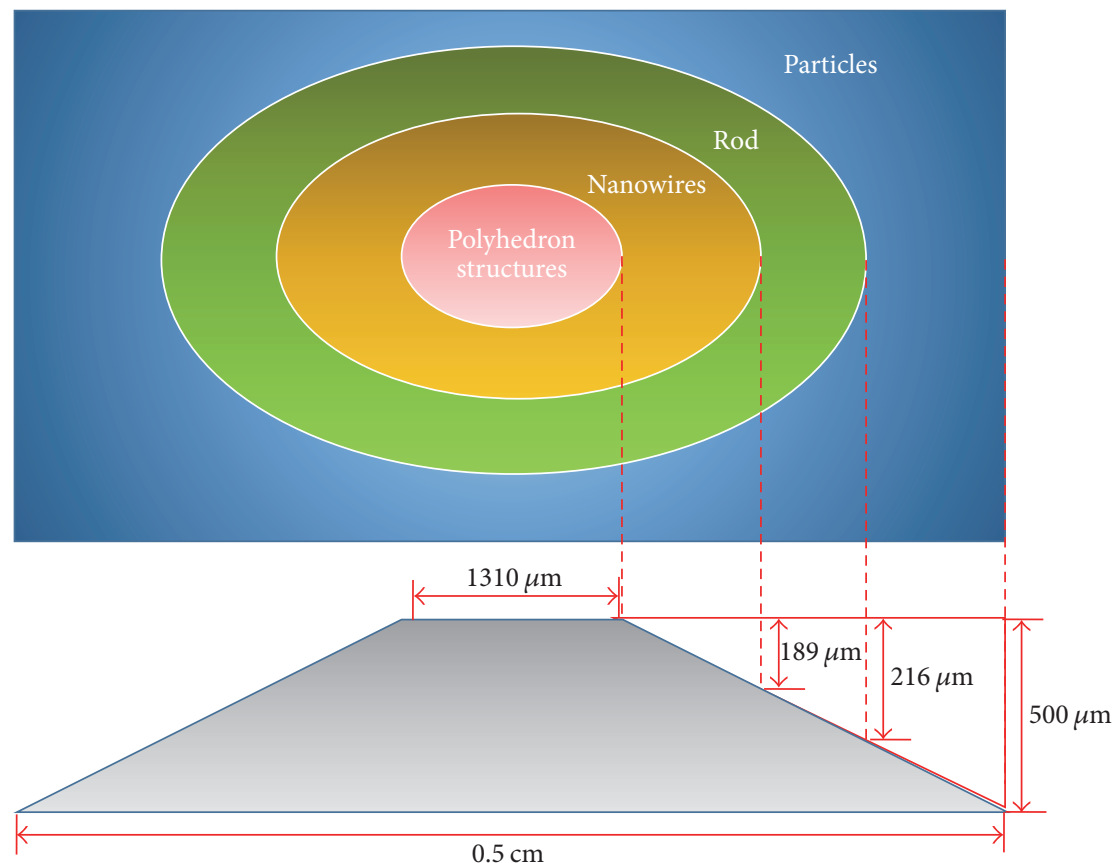

(d)

FIGURE 1: Various laying shapes of Mn powder: (a) flat, (b) cone, and (c) multiple-cones. (d) Calculation of distances from source to substrate for various products in cone laying shape of Mn powder.

(EDX) was used in this study to verify the morphology and composition of the nanostructures. Sample was attached by conductive tape on the sample holder. The voltage was adjusted from $5 \mathrm{kV}$ to $20 \mathrm{kV}$ in order to fulfill higher resolution and X- ray spectrum. X-ray diffraction (RIGAKU 2550, Japan) (XRD) was employed to confirm the primary phase of the samples with 2Theta scanning from 5 to $90^{\circ}$. Magnetic properties of the synthesized material were characterized with a magnetic property measurement system (Quantum Design MPMS3) (MPMS) capable of applying magnetic field as large as $7 \mathrm{~T}$. Cooling by liquid helium, temperature dependent magnetization was measured from 5 to $300 \mathrm{~K}$ with magnetic field set to be 1000 Oe.

\section{Results and Discussion}

To investigate the effect of the constant distance between manganese $(\mathrm{Mn})$ powder and silicon substrate on the morphology of nanostructures, Mn powder was stacked flat and smooth underneath the Mn powder (Figure 1(a)). Then two supporting silicon wafers were placed directly on the Mn powder. One silicon substrate was placed on the supporting wafer leaving a gap between substrate and powder. The gap between the substrate and Mn powder is $500 \mu \mathrm{m}$ which is the thickness of one piece of supporting wafer. Direct contact of $\mathrm{Mn}$ powder and silicon substrate was avoided. After $80 \mathrm{~min}$ deposition, the substrate was covered with a thick layer of film whose $\mathrm{Mn} / \mathrm{Si}$ composition ratio was verified to be approximately $4 / 7$ by EDX. Few Mn atoms were evaporated and captured by silicon substrate due to the longer mean free path. Forming a growth site on the surface of substrate, $\mathrm{Mn}-\mathrm{Si}$ atomic cluster began to absorb nearby silicon atoms leading to growth of Si rich phase.

Constant distance gave rise to an unchanged morphology; it was quite easier to distinguish the deposits by laying manganese powder to a cone-like shape (Figure 1(b)) in order to find the effect of various distance on the morphological evolution of $\mathrm{Mn}-\mathrm{Si}$ compounds. After deposition, four different nano/microstructures including dispersed particles, rods, nanowires, and polyhedron were found on the substrate. Each of them was growing in a ring like growing zones (Figure 2(a)) distributing from edge to center of substrate. In the center of substrate, many polyhedron structures with $10 \mu \mathrm{m}$ in width spread evenly (Figure 2(b)). Those polyhedron were a well-shaped object composed of several folding layers. The width of each layer was about hundreds of nanometers. 


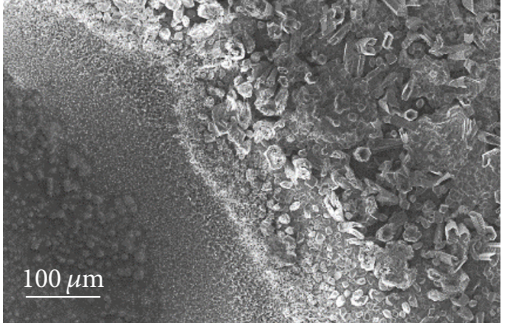

(a)

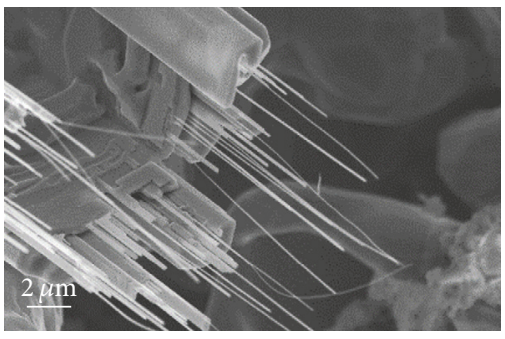

(d)

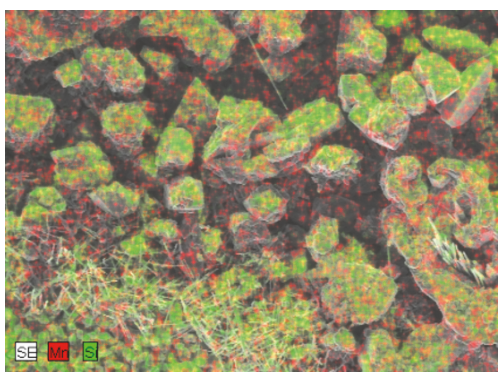

(g)

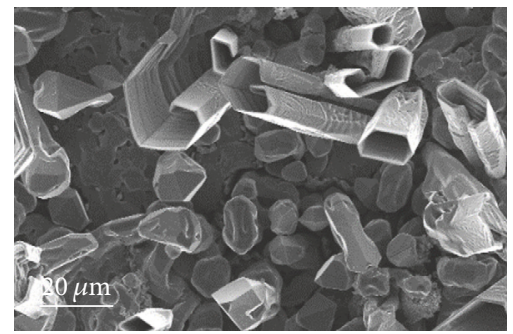

(b)

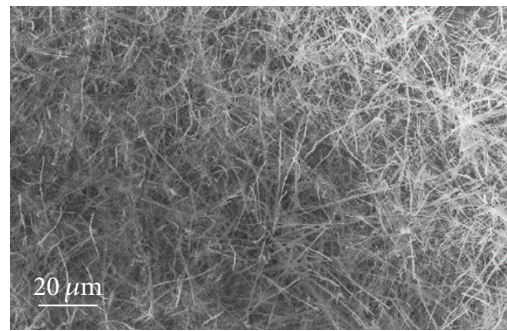

(e)



(h)

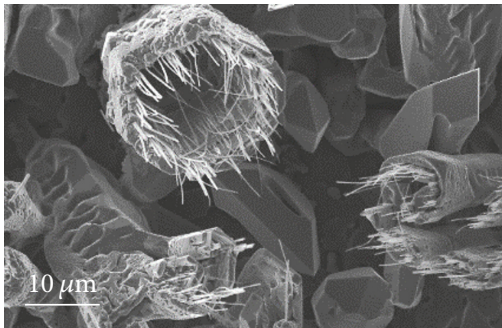

(c)

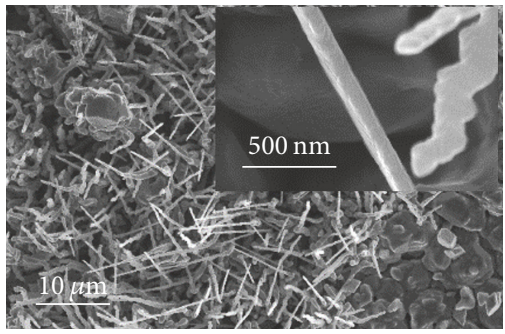

(f)

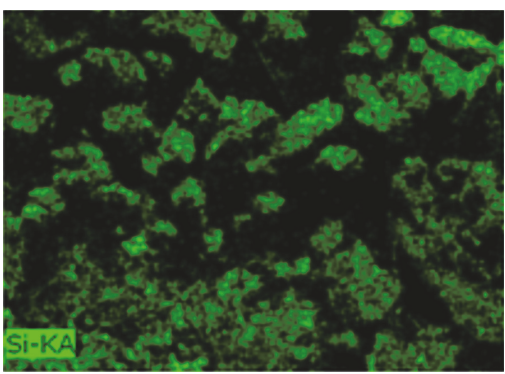

(i)

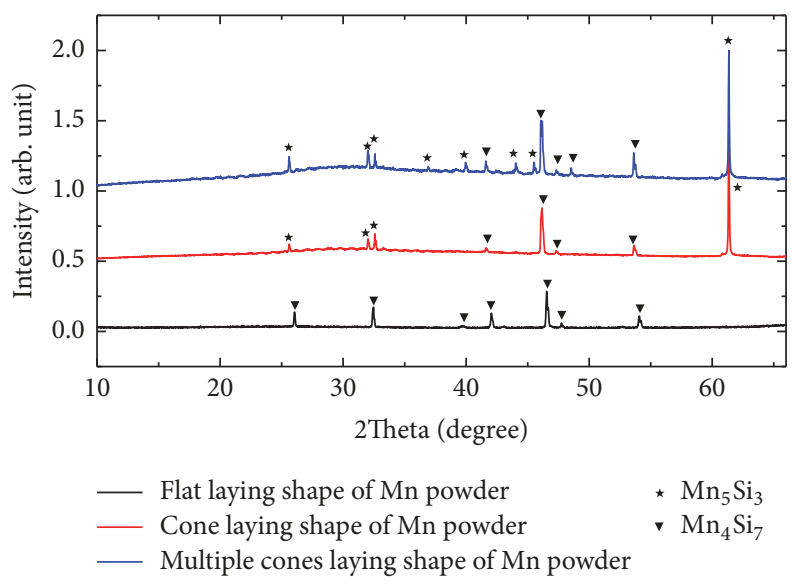

(j)

FIGURE 2: Micro/nanostructure prepared by cone laying shape of Mn powder: (a) growing bands from center to edge of substrate; (b) polyhedron structures; (c)-(d) quasi-polyhedron structures with nanowires growing in edges; (e) nanowires; (f) nanorods; (g)-(i) Mn and Si element mapping of substrate. (j) X-ray diffraction pattern of nanostructure prepared by various stacking shape of Mn powder.

In multiple-cone shape of Mn powder (Figure 1(c)), similar situation happened.

The region of growth is specialized. Considering the width of band, with the angle of bump $15.57^{\circ}$, the range of distance from substrate to powder is divided into four regions: (1) polyhedrons structures grow at area where $\mathrm{Mn}$ powder contacts with Si substrate; (2) nanowires grow at distance from $0 \mu \mathrm{m}$ to $189 \mu \mathrm{m}$; (3) nanorods grow at a narrow range of distance from $189 \mu \mathrm{m}$ to $216 \mu \mathrm{m}$; (4) particles grow at a distance from $216 \mu \mathrm{m}$ to $500 \mu \mathrm{m}$ (Figure 1(d)). The 
distance might not be that precise because in the boundary between those regions, usually two forms of nanostructure were found.

By examining the outer growing zone near polyhedron, randomly oriented nanowires were growing. Surprisingly additional quasi-polyhedron structures were prepared in the location between nanowires and polyhedron (Figure 2(c)). Those surfaces of quasi-polyhedrons were oxidized leading to several holes and inflated bumps. Quasi-polyhedrons with both features of polyhedron and nanowires revealed a clear sign of distance dependent morphological evolution. The transitional morphology was growing due to the various growth mechanisms. In this process, temperature and oxygen both played an important role. The higher concentration of $\mathrm{Mn}$ atoms was an essential condition for growth of polyhedron. The direct contact of Mn powder and silicon substrate led to the growth of polyhedrons in the area where oxygen content is relatively low. In the places very near to the center of the substrate, the distance from Mn powder to silicon substrate was so small; polyhedron firstly grew with relatively low temperature due to surrounding high concentration of $\mathrm{Mn}$ atoms. As temperature increased, polyhedron was oxidized which resulted in irregular shape forming in the surface. Then diffusion of silicon atoms was enhanced through the oxide inducing the growth of $\mathrm{Mn}-\mathrm{Si}$ nanowires.

All of them were well-shaped layered structures with very thin nanowires growing in the edges (Figure 2(d)). The nanowire was straight with tens of micrometers in length and $100-200 \mathrm{~nm}$ in width.

Nanowires found growing separately near the quasipolyhedron structures were $500-800 \mathrm{~nm}$ in width and $40-80 \mu \mathrm{m}$ in length covered with a thick layer of silicon dioxide (Figure 2(e)).

Next to nanowires band there were many nanorods distributed around (Figure 2(f)). Those nanorods were short and thinner with length of $10 \mu \mathrm{m}$ and width of $100 \mathrm{~nm}$. The distribution of nanorods was not as dense as that of nanowires. The outermost band was well-shaped particles.

In the whole surface of substrate, it was found that composition varies (Figure $2(\mathrm{~g})$ ). Element analysis was conducted by comparing only manganese Figure $2(\mathrm{~h}$ ) and silicon mapping Figure 2(i). Manganese was distributed more evenly both in the polyhedron and nanowires, while silicon was more concentrated in the surface of polyhedron.

$\mathrm{X}$-ray diffraction patterns were shown in Figure 2(j). Peaks largely depended on the laying shapes of manganese powder. With the flat laying shape of Mn powder, all the peaks matched $\mathrm{Mn}_{4} \mathrm{Si}_{7}$ solely. However, for samples growing in other two manganese powder stacking shapes, peaks of $\mathrm{Mn}_{5} \mathrm{Si}_{3}$ began to appear. The $\mathrm{Mn}_{5} \mathrm{Si}_{3}$ signals came from the polyhedron structures. Peaks became intensively distributed for more polyhedron structure resulting from larger contact areas in multiple-cones laying shape of Mn powder.

To further examine the polyhedron in the center area, atomic composition measured at five points chosen from top layer to bottom layer of the polyhedron structure was shown in Figure 3(a). Manganese mapping and silicon mapping were compared separately in Figures 3(b) and 3(c). By plotting the element concentration in Figure 3(d), composition of $\mathrm{Mn}: \mathrm{Si}$ was found at the top of polyhedron structure close to $5: 3$; then $\mathrm{Mn}: \mathrm{Si}$ atomic ratio increased to nearly $4: 1$ at the bottom of polyhedron (Figure 3(e)). Besides tiny peaks of carbon and oxygen, the EDX spectrums measured on points 1, 3, and 5 were compared in Figures 3(f), 3(g), and 3(h). It showed a gradual decrease in the height of Si peak. That means the composition of silicon dropped. Element ratio was similar to $\mathrm{Mn}$-Si chemical formula illustrating that polyhedron was multiple-phases composition gradient material.

Element mapping revealed that morphological evolution depends on whether oxygen was involved in the growth process. In the center where manganese powder directly contacts with silicon substrate, the main mechanism is a solid-solid reaction. But a layer of manganese has been found underneath the polyhedrons indicating that Mn particles touched the surface of silicon substrate forming a thin layer of high manganese compounds in absence of oxygen at low temperature; then silicon began to react with manganese to form polyhedron structure (Figure 3(e)) because EDS data showed no oxygen in the polyhedron structures.

In the border of contact area, high manganese polyhedrons began to grow at relatively low temperature first. Then as temperature increases, assisted by higher concentration of oxygen in the gap between silicon and manganese, $\mathrm{Mn}-\mathrm{Si}$ nanowires on the top of polyhedron structure began to grow. The growth in this area depended on oxygen assisted vaporsolid growth. The supersaturation ratio depending on oxygen concentration plays an important role in the composition and structure of nanowires [5].

Then in the area where Mn particle did not contact with silicon substrate, oxygen first reacted with silicon substrate at lower temperature; then when the temperature increased, $\mathrm{Mn}$ vapor directly reacted with $\mathrm{SiO}_{2}$ forming different micro/nanostructures. In this study, nanowires showed a composition ratio of $\mathrm{Mn}$ : $\mathrm{Si}$ which equals $1: 1,5: 3$, and $4: 7$ due to uneven thickness of oxide forming on silicon substrate.

By applying magnetic field ( 1000 oe) parallel to the substrate surface, it is clear that various Mn powder stacking shape resulted in different magnetic signals (Figure 4). It is obvious that black curve was smooth and has only one transition near $40 \mathrm{~K}$ which corresponds to X-ray pattern showing mostly $\mathrm{Mn}_{4} \mathrm{Si}_{7}$ on sample. When $\mathrm{Mn}$ powder was stacking in a shape with one bump, the manganese element will be deposited on the substrate in shorter time with less oxygen involved. So $\mathrm{Mn}-\mathrm{Si}, \mathrm{Mn} 4 \mathrm{Si}$, and $\mathrm{Mn}_{5} \mathrm{Si}_{3}$ nanowires contributed to all signals. The relative amount of $\mathrm{Mn}_{4} \mathrm{Si}_{7}$ was reduced leading to a weaker magnetic signal of $40 \mathrm{~K}$ transition point (Figure 4 inset (a)). In Figure 4 inset (b), two new transitions were observed near $75 \mathrm{~K}$ and $220 \mathrm{~K}$. Sample prepared with Mn powder stacking in several cones shows stronger ferromagnetic signal near $220 \mathrm{~K}$ than that prepared with $\mathrm{Mn}$ powder stacking into one cone, while on the contrary, sample prepared with Mn powder stacking in several cones shows weaker antiferromagnetic signal near the transitions at approximately $75 \mathrm{~K}$ than that prepared with Mn powder piling up with one cone. The antiferromagnetic transition point at $75 \mathrm{~K}$ relies on the $\mathrm{Mn}_{5} \mathrm{Si}_{3}$ nanowires and bulk $\mathrm{Mn}_{5} \mathrm{Si}_{3}$ which came from the area where Mn powder 


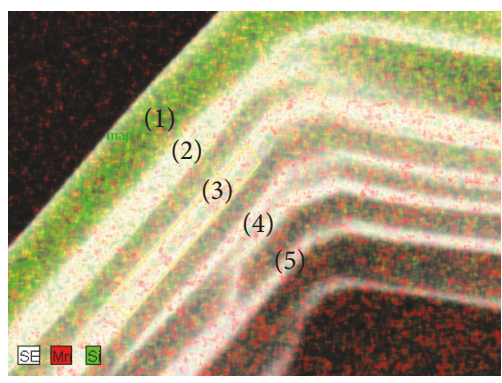

(a)

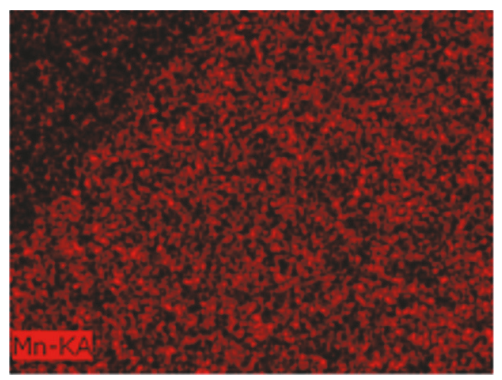

(b)

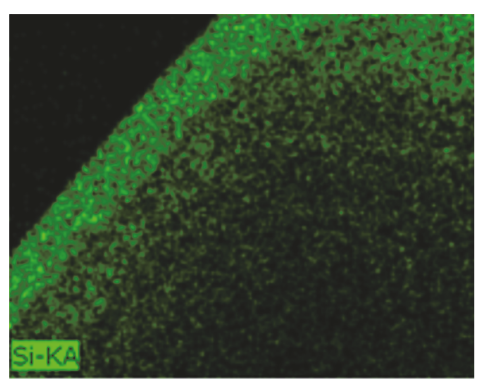

(c)
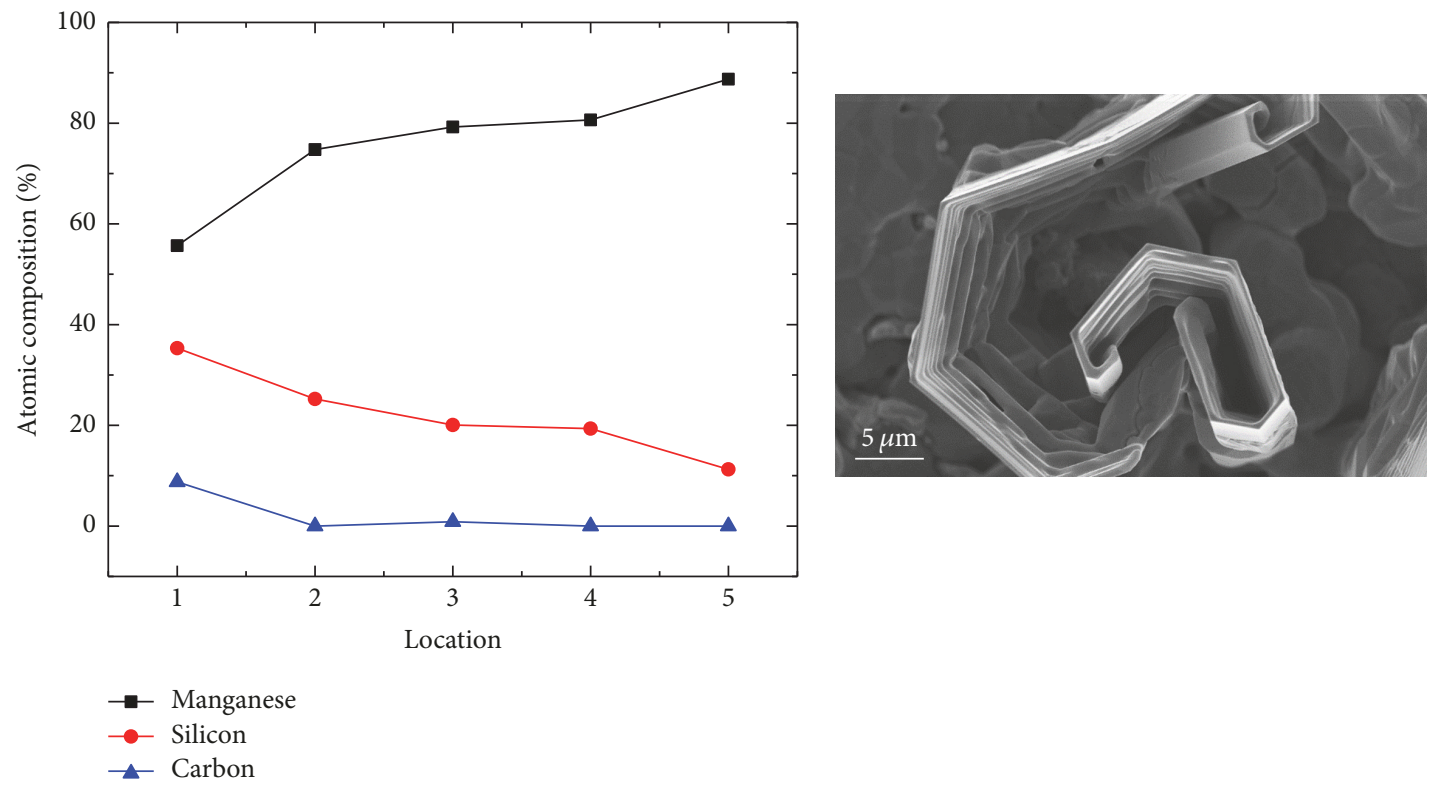

(d)

(e)

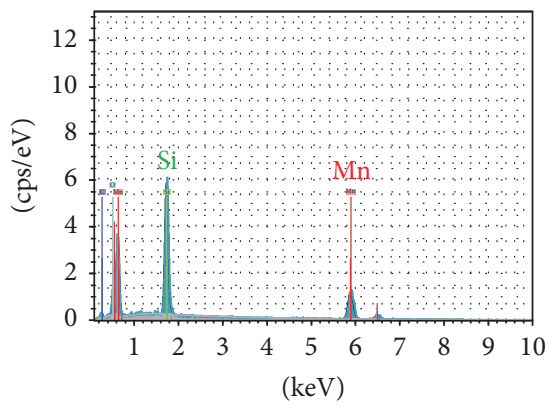

(f)

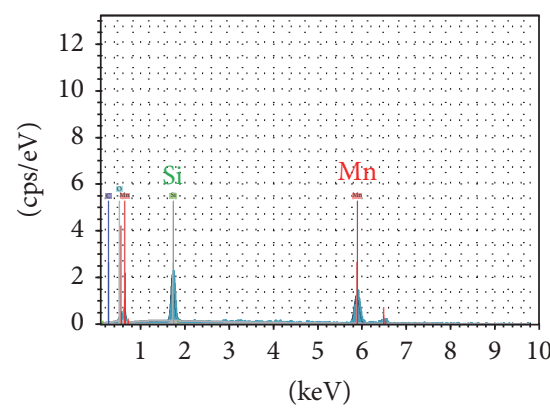

(g)

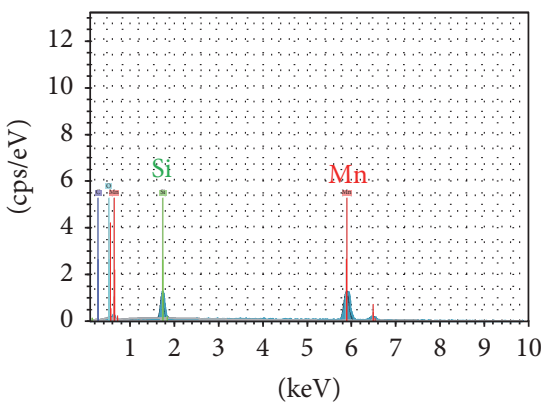

(h)

FIGURE 3: (a)-(c) Mn and Si element mapping of composition graded polyhedron materials prepared by direct reaction of Mn particles with silicon wafer; (d) change of $\mathrm{Mn}$ and Si concentration via location points from top to down; (e) typical polyhedron structures; (f)-(h) measured spectrum of elements $\mathrm{Mn}$ and $\mathrm{Si}$ at points 1,3 , and 5.

contacts with silicon substrate. Another ferromagnetic transition point at 220-250 K relies on the diluted magnetic semiconductor $\mathrm{Mn}_{x} \mathrm{Si}_{1-x}(x=0.03-0.05)$. This diluted magnetic semiconductor form basing on $\mathrm{Mn}$ atom was incorporated into the silicon lattice. Liu et al. prepared this material by sputtering single crystalline Si partially covered with Mn chips on silicon substrate $[15,16]$. So it is estimated that $\mathrm{Si}$ contacting with $\mathrm{Mn}$ chips directly might be the reason for preparing this diluted magnetic semiconductor successfully. Thus in sample prepared with stacking Mn powder in several bumps, more Mn-Si contact points brought about more $\mathrm{Mn}_{x} \mathrm{Si}_{1-x}(x=0.03-0.05)$ leading to stronger ferromagnetic signal at $220 \mathrm{~K}$.

\section{Conclusion}

Direct contact of $\mathrm{Mn}$ powder and silicon substrate gave rise to the solid-solid reaction of $\mathrm{Mn}$ and $\mathrm{Si}$, leading to the growth of polyhedrons (a special type of chemical composition gradient functional graded materials). Nanowires, nanorods, 


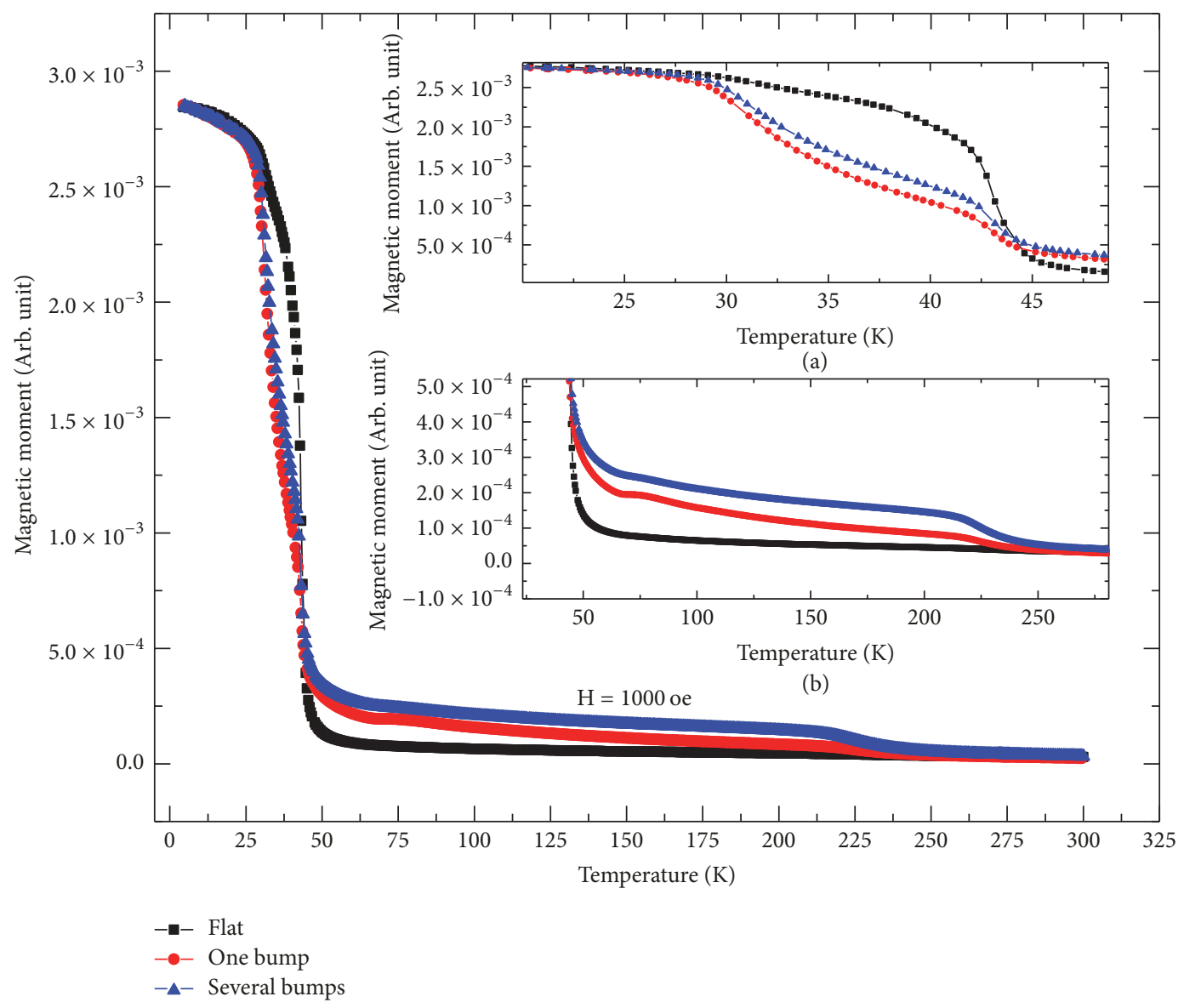

FIGURE 4: Magnetic properties showing various transition temperatures correlated to different nano/microphases. Inset figures magnetic transitions in the temperature ranges (a) $20-50 \mathrm{~K}$; (b) 50-300 K.

and particles were prepared by oxygen assisted vapor-solid growth on the area where Mn particle did not contact with Si wafer. The spatial morphological evolution influenced by distance from precursor to substrate clearly revealed trend of varying the supersaturation ratio. Finally, magnetic signal not only shows transitions of $\mathrm{Mn}-\mathrm{Si}, \mathrm{Mn}_{4} \mathrm{Si}_{7}$, and $\mathrm{Mn}_{5} \mathrm{Si}_{3}$, but also gives a ferromagnetic signal of diluted magnetic semiconductor $\mathrm{Mn}_{x} \mathrm{Si}_{1-x}(x=0.03-0.05)$.

\section{Conflicts of Interest}

The author declares that there are no conflicts of interest regarding the publication of this paper.

\section{References}

[1] T. Itoh and M. Yamada, "Synthesis of thermoelectric manganese silicide by mechanical alloying and pulse discharge sintering," Journal of Electronic Materials (JEM), vol. 38, no. 7, pp. 925-929, 2009.

[2] A. L. Schmitt, N. M. Ahmad, J. M. Higgins, J. R. Szczech, and S. Jin, "Synthesis and applications of metal silicide nanowires," Journal of Materials Chemistry, vol. 20, pp. 223-235, 2010.

[3] S. Mühlbauer, B. Binz, F. Jonietz et al., "Skyrmion lattice in a chiral magnet," Science, vol. 323, no. 5916, pp. 915-919, 2009.
[4] J. M. Higgins, R. Ding, J. P. Degrave, and S. Jin, "Signature of helimagnetic ordering in single-crystal MnSi nanowires," Nano Letters, vol. 10, no. 5, pp. 1605-1610, 2010.

[5] S. Tang, I. Kravchenko, J. Yi et al., "Growth of skyrmionic MnSi nanowires on Si: Critical importance of the $\mathrm{SiO} 2$ layer," Nano Research, vol. 7, no. 12, pp. 1788-1796, 2014.

[6] A. Mehlin, F. Xue, D. Liang et al., "Stabilized Skyrmion Phase Detected in MnSi Nanowires by Dynamic Cantilever Magnetometry," Nano Letters, vol. 15, no. 7, pp. 4839-4844, 2015.

[7] X. Z. Yu, N. Kanazawa, W. Z. Zhang et al., "Skyrmion flow near room temperature in an ultralow current density," Nature Communications, vol. 3, pp. 1-6, 2012.

[8] S. Teichert, H. Hortenbach, and H.-J. Hinneberg, "Surfactant mediated growth of MnSil.7 on Si(001)," Applied Physics Letters, vol. 78, no. 14, pp. 1988-1990, 2001.

[9] M.-H. Ham, J.-W. Lee, K.-J. Moon, J.-H. Choi, and J.M. Myoung, "Single-crystalline ferromagnetic Mn 4Si 7 nanowires," The Journal of Physical Chemistry C, vol. 113, no. 19, pp. 8143-8146, 2009.

[10] U. Gottlieb, A. Sulpice, B. Lambert-Andron, and O. Laborde, "Magnetic properties of single crystalline Mn4Si7," Journal of Alloys and Compounds, vol. 361, no. 1-2, pp. 13-18, 2003.

[11] H. Liu, G. She, X. Huang et al., "Synthesis and magnetic properties of $\mathrm{Mn} 4 \mathrm{Si} 7$ and $\mathrm{Si}-\mathrm{Mn} 4 \mathrm{Si} 7$ axial heterostructure 
nanowire arrays," The Journal of Physical Chemistry C, vol. 117, no. 5, pp. 2377-2381, 2013.

[12] B. Das, B. Balasubramanian, P. Manchanda et al., "Mn5Si3 Nanoparticles: Synthesis and Size-Induced Ferromagnetism," Nano Letters, vol. 16, no. 2, pp. 1132-1137, 2016.

[13] J. Leciejewicz, B. Penc, A. SzytuŁa, A. Jezierski, and A. Zygmunt, "Magnetic properties of the Mn5Si3 compound," Acta Physica Polonica A, vol. 113, no. 4, pp. 1193-1203, 2008.

[14] P. J. Brown and J. B. Forsyth, "Antiferromagnetism in Mn5Si3: The magnetic structure of the AF2 phase at $70 \mathrm{~K}$," Journal of Physics: Condensed Matter, vol. 7, no. 39, pp. 7619-7628, 1995.

[15] X. C. Liu, Z. H. Lu, Z. L. Lu et al., "Hole-mediated ferromagnetism in polycrystalline Sil-xMnx:B films," Journal of Applied Physics, vol. 100, no. 7, article 073903, pp. 1-5, 2006.

[16] X. C. Liu, Y. B. Lin, J. F. Wang et al., "Effect of hydrogenation on the ferromagnetism in polycrystalline Sil-x Mnx: B thin films," Journal of Applied Physics, vol. 102, no. 3, pp. 1-4, 2007.

[17] M. Hortamani, L. Sandratskii, P. Zahn et al., "Physical origin of the incommensurate spin spiral structure in Mn3Si," Journal of Applied Physics, vol. 506, pp. 10-13, 2012.

[18] C. Pfleiderer, "Stability of antiferromagnetism at high magnetic fields in Mn3Si," Physical Review B: Condensed Matter and Materials Physics, vol. 65, pp. 1-4, 2002.

[19] F. Takano, H. Akinaga, H. Ofuchi, S. Kuroda, and K. Takita, "Characterization of room temperature ferromagnetic Mn-Si compound synthesized on SiC substrate," Journal of Applied Physics, vol. 99, no. 8, pp. 1-4, 2006. 


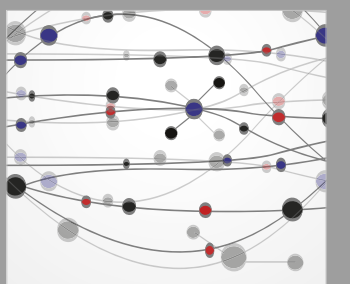

The Scientific World Journal
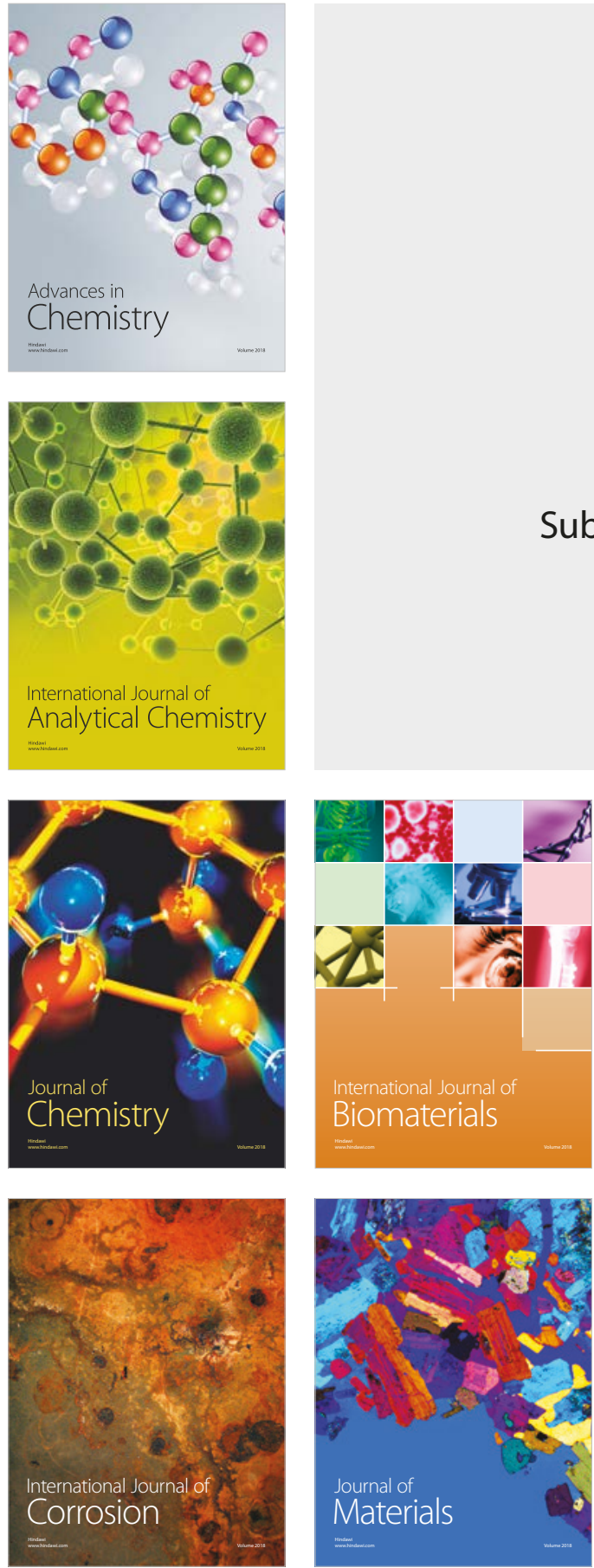

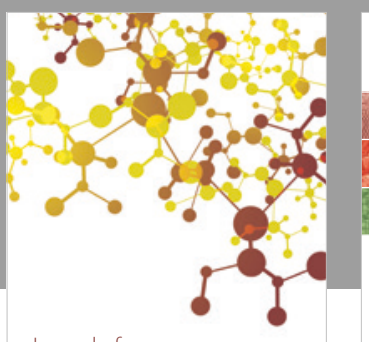

Journal of

Applied Chemistry
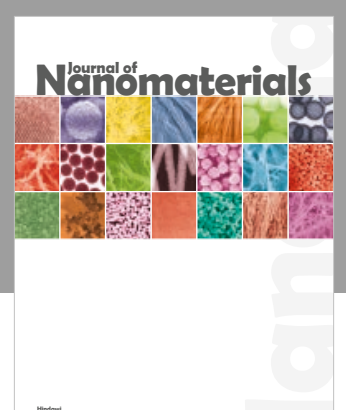

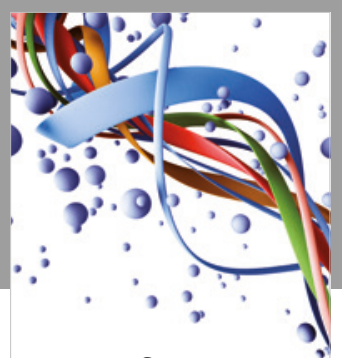

Scientifica

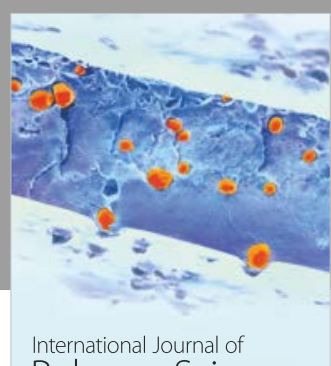

Polymer Science

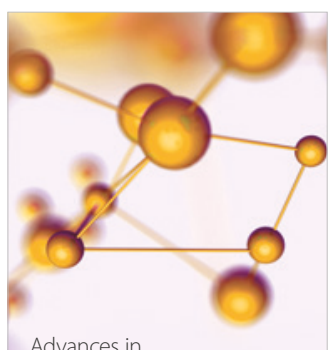

Physical Chemistry
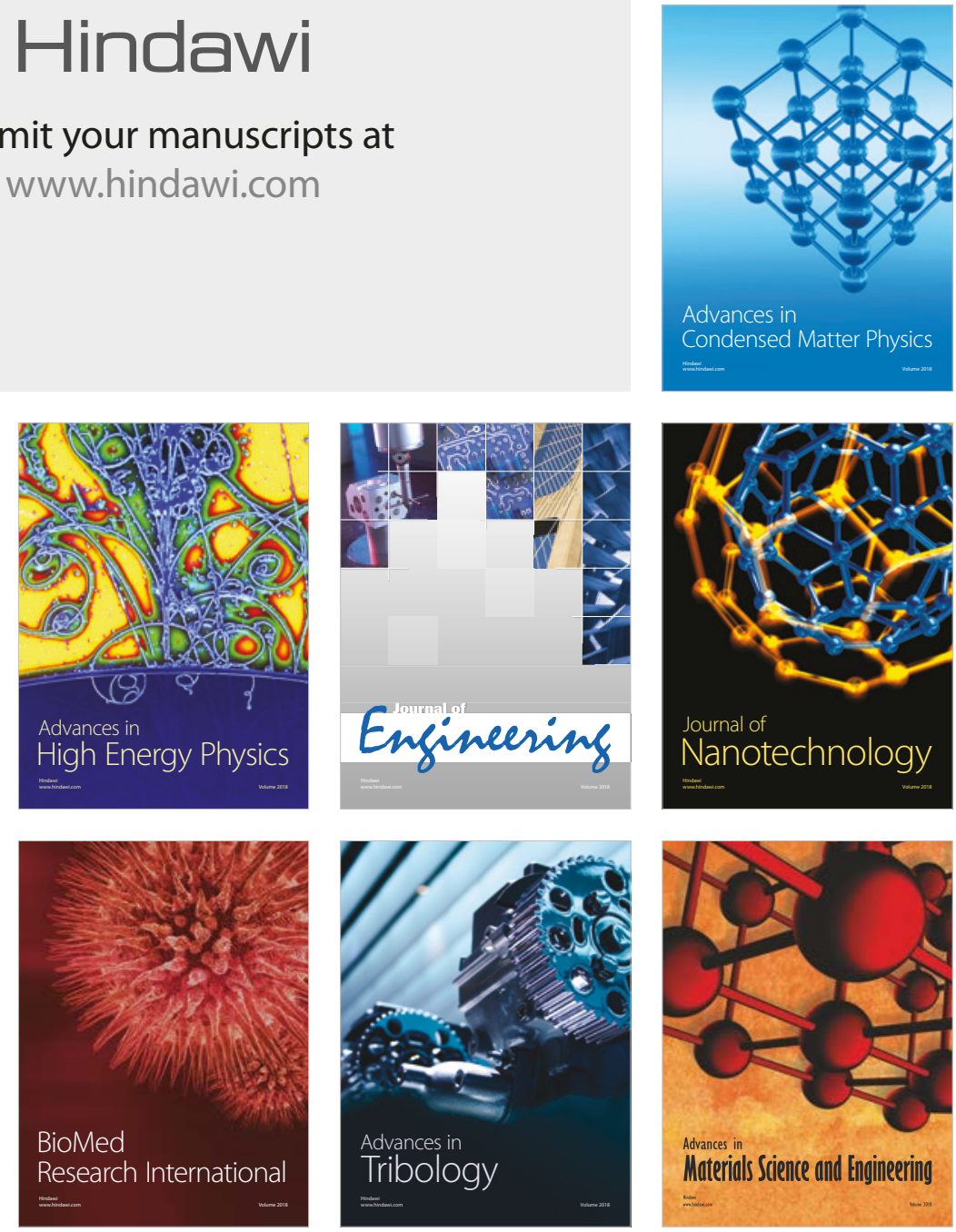